\title{
Classification of Satellite Images by means of Fuzzy Rules generated by a Genetic Algorithm
}

\author{
O. Gordo, E. Martínez, C. Gonzalo and A. Arquero
}

\begin{abstract}
The data acquired by Remote Sensing systems allow obtaining thematic maps of the earth's surface, by means of the registered image classification. This implies the identification and categorization of all pixels into land cover classes. Traditionally, methods based on statistical parameters have been widely used, although they show some disadvantages. Nevertheless, some authors indicate that those methods based on artificial intelligence, may be a good alternative. Thus, fuzzy thematic classifiers, which are based on Fuzzy Logic, include additional information in the classification process through based-rule systems. In this work, we propose the use of a genetic algorithm (GA) to select the optimal and minimum set of fuzzy rules to classify remotely sensed images by means a fuzzy classifier. Input information of GA has been obtained through the training space determined by two uncorrelated spectral bands (2D scatter diagrams), which has been irregularly divided by five linguistic terms defined in each band. The proposed methodology has been applied to Landsat-TM images and it has showed that this set of rules provides a higher accuracy level in the classification results.
\end{abstract}

Keywords - Fuzzy thematic classifier, fuzzy rules, genetic algorithm, remotely sensed images.

\section{INTRODUCCIÓN}

$\mathrm{L}^{\prime}$ A Teledetección se muestra como una buena fuente de información sobre los fenómenos que ocurren en las cubiertas terrestres. A partir de ella se pueden obtener, con un alto grado de exactitud, mapas temáticos de la superficie terrestre a diferentes escalas espacio-temporales. Sin embargo, no es sencillo extraer información precisa sobre los diferentes tipos de cubiertas presentes en ella, a partir de datos remotamente detectados. Esta tarea se ve influenciada por diferentes factores, como los efectos atmosféricos; las características de los sensores: resolución espacial, espectral, temporal y radiométrica; y básicamente por la metodología seleccionada para generar el mapa de clases (clasificador). La clasificación es esencialmente un problema de reconocimiento de patrones [1] y juega un papel importante en el análisis de la información obtenida mediante sistemas de Teledetección [2].

O. Gordo, Universidad Politécnica de Madrid (UPM), Madrid, España, oscar.gordo@bull.es

E. Martínez, Universidad Politécnica de Madrid (UPM), Madrid, España, emartinez@fi.upm.es

C. Gonzalo, Universidad Politécnica de Madrid (UPM), Madrid, España, onsuelo.gonzalo@upm.es

A. Arquero, Universidad Politécnica de Madrid (UPM), Madrid, España, aarquero@fi.upm.es
El proceso normalmente implica la identificación y categorización de todos los píxeles de la imagen en clases de terreno. Cada una de estas categorías es asociada con un patrón espectral (firma espectral) que es utilizado como base numérica de la categorización.

El objetivo de este trabajo ha sido desarrollar una metodología de clasificación, mediante la utilización de un clasificador temático difuso, que utiliza como base de conocimiento, información espectral procedente de diagramas de dispersión en dos dimensiones (2D). La utilización de estos diagramas supone una mejora en la generación no subjetiva de conocimiento para el clasificador.

\section{ANTECEDENTES}

La literatura tipifica los clasificadores según diversos criterios. Clásicamente se describen dos tipos de procedimientos de clasificación: supervisados y no supervisados [3]. En el caso de los no supervisados, los datos de la imagen son clasificados mediante agrupamientos basados en similitud espectral, sin que exista un proceso de etiquetado mediante supervisión de campo. En los supervisados, el clasificador es entrenado mediante descriptores numéricos de las clases que son ejemplos representativos de clases temáticas de terreno. Cada ejemplo es conocido como área de entrenamiento y cada píxel de la imagen es comparado numéricamente con los parámetros obtenidos de dichas áreas.

Otro criterio a considerar, es la dependencia de los clasificadores de parámetros estadísticos, como es el caso del clasificador de mínima distancia o el de máxima verosimilitud que han sido los utilizados con mayor frecuencia. Estos métodos tienen sus limitaciones, particularmente en relación con la asunción de que los patrones de entrenamiento se comportan según una distribución Gaussiana [4]. Sin embargo, en algunas ocasiones esto no ocurre y la distribución no es normal. Recientemente numerosos estudios han demostrado que metodologías basadas en inteligencia artificial, como sistemas expertos, redes neuronales o máquinas de soporte vectorial [5], resuelven algunos problemas inherentes a las metodologías basadas en parámetros estadísticos. Los sistemas expertos o métodos basados en conocimiento son caracterizados por dos componentes: la base de conocimiento y el motor de inferencia [6]. El "cuello de botella" de los sistemas expertos es el problema de extracción de conocimiento, debido a que se requiere bastante tiempo en desarrollar la base de reglas [4] [7].

Un tercer criterio para agrupar clasificadores nace en la 
consideración de que los clasificadores clásicos, solo usan lógica bimodal, indicando que cada píxel pertenece o no pertenece a una clase particular. Estos utilizan valores numéricos con poco significado, y no utilizan información heurística. En la práctica, la presencia de zonas de heterogeneidad espectral en la imagen, puede conducir a resultados de clasificación poco satisfactorios. Otros clasificadores, como los difusos o basados en lógica difusa [8] [9], incluyen información adicional en el proceso de clasificación mediante sistemas basados en reglas. Son una alternativa eficaz a las dificultades comentadas anteriormente. En un sistema de clasificación difusa [10], los objetos pueden ser clasificados mediante la aplicación de una serie de reglas basadas en unos términos lingüísticos y en sus atributos. Es precisamente de nuevo, la generación de un conjunto de reglas, el paso crítico en el proceso de clasificación. Estas reglas se pueden generar de manera subjetiva por un experto humano, o de manera automática. Por lo tanto, resulta muy útil disponer de algoritmos que permitan realizar esta tarea de adquisición de conocimiento, de manera objetiva y automática [11]. Actualmente, algunos autores [12] ya han utilizado los sistemas basados en reglas obtenidas a partir de algoritmos genéticos para clasificar imágenes multiespectrales. Sin embargo, la aplicación conjunta de algoritmos genéticos y sistemas difusos en el ámbito de la Teledetección, solo se ha realizado en el caso de clasificadores neurodifusos [13] [14]. Los métodos difusos están orientados principalmente a la adquisición de reglas difusas tipo IF-THEN que se traducen a código binario y conjuntos difusos (funciones miembro). Una de sus ventajas es que la adquisición se hace a partir de datos numéricos, por lo que el método es especialmente útil en dominios en los que no existe un experto o bien éste no tiene un conocimiento sobre él.

Los algoritmos genéticos son algoritmos evolutivos que pertenecen a un grupo de técnicas estocásticas que utilizan los conceptos de evolución biológica. Actúan sobre una población de soluciones potenciales aplicando los principios de diversidad de individuos y supervivencia del más fuerte para producir mejores aproximaciones a una solución [15]. La idea subyacente en los algoritmos genéticos de adquisición de datos difusos, es la obtención de un conjunto de reglas óptimo: mínimo número de reglas que resuelvan el problema que se esté tratando, mediante la aplicación de los operadores genéticos [14] [16]: mutación y entrecruzamiento.

En este trabajo, se plantea la adaptación de un algoritmo genético propuesto por Yuan et al. [17] para determinar el conjunto óptimo de reglas difusas para su aplicación a la clasificación de imágenes remotamente detectadas.

\section{DESCRIPCIÓN DEL SISTEMA DE CLASIFICACIÓN DIFUSA}

El proceso de construcción del sistema de clasificación difusa se inicia con la generación de reglas y reducción de la base de reglas mediante algoritmos genéticos. Posteriormente se aplica el método de clasificación a nuevos patrones con las reglas adquiridas, lo que constituye la inferencia del modelo.

La información de partida para el desarrollo de este proceso, la constituye un conjunto de patrones de entrenamiento etiquetados de acuerdo a las clases estimadas para los elementos del dominio en estudio. Asimismo se deben establecer los atributos, sus términos lingüísticos (valores cualitativos) y las funciones miembro (valores cuantitativos) que constituyen las particiones del dominio de aplicación de la metodología.

\section{A. Algoritmo genético para la generación de reglas difusas en clasificación multiespectral temática}

El objetivo prioritario es encontrar un conjunto compacto de reglas con una alta eficiencia en la clasificación temática. Este se puede desglosar en la consecución de dos objetivos parciales:

1) Maximización del número de los patrones de entrenamiento bien clasificados por el conjunto de reglas.

2) Minimización del número de reglas del conjunto.

En la Fig. 1 se muestra el diagrama que representa el algoritmo de generación del conjunto de reglas que debe cumplir los objetivos enunciados anteriormente.

En primer lugar, se define y codifica un conjunto inicial de reglas. La experiencia demuestra que un pequeño porcentaje deben ser de origen aleatorio [17] y el resto se pueden construir a partir de los patrones de entrenamiento. Cada regla se codifica con una cadena de bits a la que se la denomina "cromosoma".

A partir del conjunto inicial, se extraen las reglas obtenidas como consecuencia de una evaluación previa realizada mediante una función Fitness y tres parámetros denominados nivel de significación $(\alpha)$, nivel de precisión $(\beta)$ y nivel de tolerancia $(\delta)$ que conforman el criterio de parada [17]. El significado de la función Fitness, dentro del marco de los algoritmos genéticos, es el de proporcionar el estado de "salud" de cada regla o individuo de la población que se esté evaluando. Así puede ser utilizado en la reproducción con otros individuos (padres) para obtener descendientes (hijos), o simplemente eliminado.

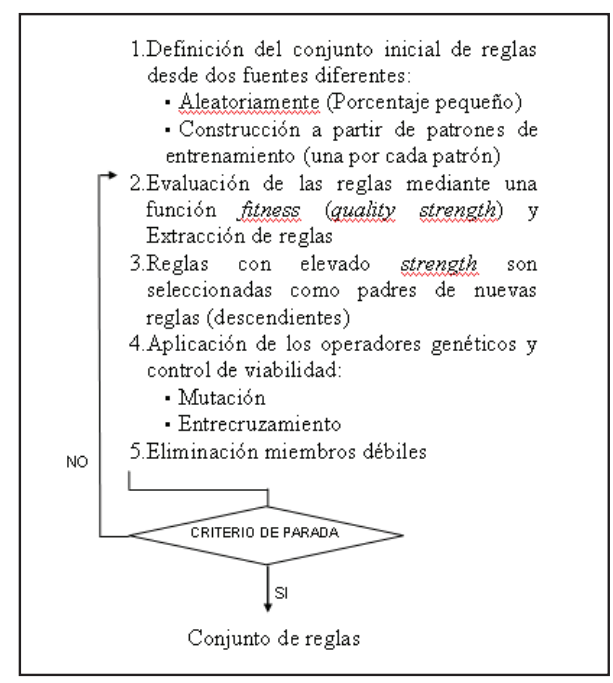

Figura 1. Diagrama para la generación de reglas difusas. 
En el proceso se aplican para la generación de reglas descendientes, los operadores genéticos siguientes:

a) Operador Entrecruzamiento. Se generan dos nuevos cromosomas a partir del intercambio de un segmento entre dos cromosomas ya existentes.

b) Operador Mutación. Se genera un nuevo cromosoma mediante la modificación de uno o más segmentos de un cromosoma ya existente.

La Fig. 2 ilustra el comportamiento del operador entrecruzamiento.

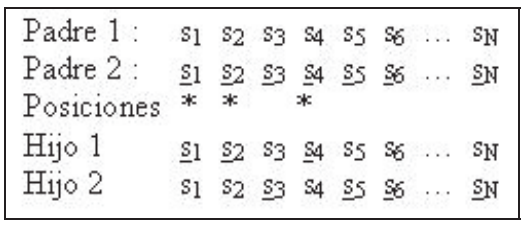

Figura 2. Entrecruzamiento.

El cromosoma denominado Hijo 1 se obtiene al cambiar las posiciones 1, 2 y 4 del cromosoma Padre 1 por las del Padre 2, y el Hijo 2 al cambiar estas mismas posiciones en el Padre 2 por las del Padre 1.

Para cada hijo generado en el entrecruzamiento, se aplica la operación de mutación de acuerdo a las siguientes reglas:

$\mathrm{S}_{\mathrm{r}}=1 \rightarrow \mathrm{S}_{\mathrm{r}}=-1$ con una probabilidad de mutación $\mathrm{P}_{\mathrm{m}}(1 \rightarrow-1)$

$\mathrm{S}_{\mathrm{r}}=-1 \rightarrow \mathrm{S}_{\mathrm{r}}=1$ con una probabilidad de mutación $\mathrm{P}_{\mathrm{m}}(-1 \rightarrow 1)$

En ambos casos la probabilidad de mutación es la misma, aunque no tendría porque ser así en el caso general.

\section{B. Inferencia del modelo}

La inferencia del modelo se basa en la aplicación del Modus Ponens Difuso que consiste en partir de una regla definida bajo los supuestos de la lógica difusa y aplicar el proceso de deducción contenido en la regla a un conjunto de datos de entrada o patrones de entrenamiento [19]. La inferencia aplicada a la clasificación de patrones es un caso particular del Modus Ponens Difuso, donde la diferencia fundamental es que los patrones se definen sobre valores numéricos y no sobre términos lingüísticos. De esta forma, el consecuente de la regla está constituido por una clase.

Como se puede observar en la Fig3 se ilustra la inferencia del modelo con un ejemplo de clasificación.

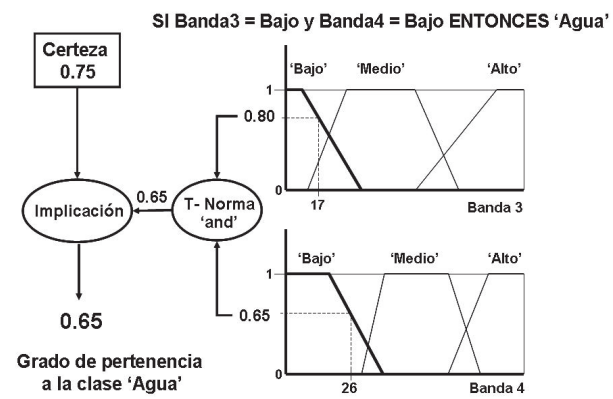

Figura 3. Ejemplo de aplicación del Modus Ponens Difuso al problema de clasificación de patrones.

\section{Metodología}

\section{A. Zona de estudio. Información sobre las clases}

Una vez seleccionada la imagen que se va clasificar, se realiza una visualización previa mediante una composición color RGB para discriminar las posibles clases de cobertura de terreno que existen. En ocasiones es conveniente consultar información cartográfica o realizar visitas in situ.

\section{B. Conjunto de áreas de entrenamiento y funciones miembro}

La selección de las áreas de entrenamiento constituye un proceso crítico pues son un estimador de las clases temáticas finales. En este trabajo, esta selección se ha llevado a cabo mediante la utilización de diagramas de dispersión en dos dimensiones [18]. El conjunto de entrenamiento es obtenido a partir del dominio espectral 2D definido por los valores en dos bandas no correladas, para todos los píxeles de la imagen. El espacio de entrenamiento, se puede definir mediante la generación de "nubes de puntos" o agrupamientos homogéneos que representan una determinada clase espectral $\mathrm{y}$ que pueden ser localizados en el dominio espacial (máscaras), seleccionando así los patrones de entrenamiento. Este procedimiento reduce en gran manera la subjetividad que implica la manera tradicional de la elección de áreas de entrenamiento sobre la imagen por parte de un humano.

El conocimiento difuso es adquirido desde el espacio de entrenamiento que emplea los valores espectrales en las dos bandas del dominio espectral como atributos. Para cada atributo pueden ser definidos muchos términos lingüísticos; sin embargo, estudios previos indican que un número impar [11] de términos trapezoidales que dividan el dominio; conduce a una buena adquisición de conocimiento. Los términos lingüísticos se definen mediante cuatro valores $\left(a_{i}, b_{i}\right.$, $\mathrm{c}_{\mathrm{i}}, \mathrm{d}_{\mathrm{i}}$ ) para cada atributo $\mathrm{B}_{\mathrm{i}}$ como se muestra en la Fig4. La correcta construcción de estas funciones es básica para el buen funcionamiento del algoritmo, ya que si no puede que haya ejemplos que nunca se clasifiquen bien o incluso que desaparezcan todas las reglas de una misma especie.

En primer lugar, se determinan las bandas que proporcionan la información necesaria, que en el ejemplo considerado han sido la Banda 3 y la Banda 4, bandas que constituyen los atributos de la regla. Para cada una de ellas se han definido tres términos lingüísticos o funciones miembro: bajo, medio y alto. Finalmente para acabar con la construcción de los elementos necesarios en el modelo, se define una regla (SI Banda3=Bajo y Banda4=Bajo ENTONCES 'Agua'), así como las funciones de composición de predicados, necesarias para obtener el nivel de veracidad del antecedente de la regla (T-norma) y el grado de pertenencia a la clase que se muestra en el consecuente (implicación), en función de la certeza asociada la regla y el valor deducido del antecedente de la misma. Para este ejemplo se ha elegido la función mínimo en ambos casos. 


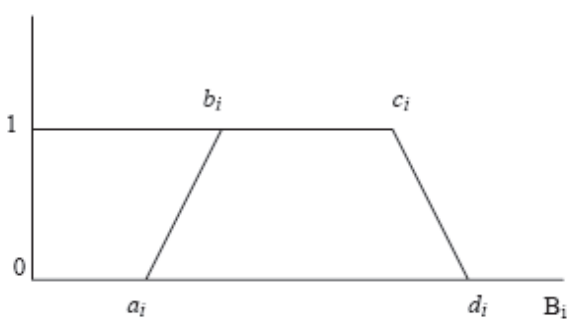

Figura 4. Representación gráfica de un término lingüístico.

Una vez definido el modelo se aplica el proceso de inferencia. Así, si se recibe un patrón a clasificar, con un valor de 17 en la Banda 3 y 26 en la Banda 4, y se aplican las funciones miembro establecidas en el antecedente de la regla: función bajo para la Banda 3 y función bajo para la Banda 4 .

Los dos valores obtenidos se componen mediante la Tnorma elegida (mínimo en este caso) y se obtiene un valor de veracidad de 0.65. Este valor se asocia al antecedente de la regla y se aplica la función de implicación (el mínimo en este caso), obteniendo un valor de 0.65 como grado de pertenencia del píxel o patrón a la clase agua. Otra lectura del resultado de este ejemplo es: "La probabilidad de que el píxel cuyos valores en bandas 3 y 4 son 17 y 26 respectivamente, pertenezca a la clase agua es del 65\%".

\section{RESUltados}

La imagen multiespectral utilizada para este estudio es una escena de 384x384 píxeles, registrada por el sensor Landsat TM situada geográficamente en los alrededores de Madrid (España). Se utilizan las bandas TM1-TM5 y TM7 de la misma resolución espacial $(30 \times 30 \mathrm{~m})$. Se han supervisado in situ siete clases temáticas: la clase agua correspondiente a un pantano cercano, tres tipos de vegetación como robledales (bosque caduco), matorral y pinar; dos tipos de suelo y una clase mixta. Los dos tipos de suelo corresponden a un suelo más reflectante denominado suelo claro, debido a composiciones graníticas y a un suelo menos reflectante denominado suelo oscuro. En el caso de la clase mixta, se trata de una clase con un comportamiento espectral heterogéneo intermedio entre una cubierta de suelo y vegetación. En la Fig5 se muestra la gráfica correspondiente al diagrama de dispersión 2D entre las bandas TM3 (B3) y TM4 (4) de dicha imagen. En este dominio espectral se han remarcado los polígonos que determinan los agrupamientos de píxeles con similitud espectral de las clases temáticas supervisadas. A partir de los valores de B3 y B4 que determinan los rectángulos, se generan las máscaras espectrales sobre la imagen que permitirán seleccionar los patrones de entrenamiento en el dominio espacial [18] [20].

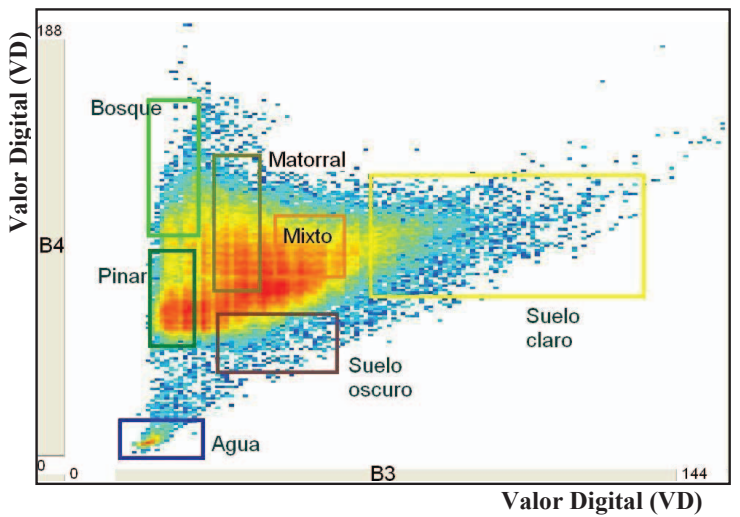

Figura 5. Diagrama de dispersión 2D para las bandas B3 y B4.

TABLA I: FUNCIONES MIEMBRO CADA TÉRMINO LINGÜÍSTICO

\begin{tabular}{|c|c|}
\hline $\begin{array}{c}\text { TERMINOS } \\
\text { LINGUISTICOS }\end{array}$ & $\begin{array}{c}\text { FUNCIONES MIEMBRO } \\
\mathbf{a}_{\mathbf{i}}, \mathbf{b}_{\mathbf{i}}, \mathbf{c}_{\mathbf{i}}, \mathbf{d}_{\mathbf{i}} \mathbf{( V D )}\end{array}$ \\
\hline \multicolumn{2}{|c|}{ B3 } \\
\hline Muy Grande & $50,57,255,255$ \\
\hline Grande & $42,43,49,50$ \\
\hline Mediano & $30,32,42,44$ \\
\hline Pequeño & $18,20,28,30$ \\
\hline Muy Pequeño & $0,0,19,20$ \\
\hline \multicolumn{2}{|c|}{ B4 } \\
\hline Muy Grande & $116,120,255,255$ \\
\hline Grande & $96,100,116,120$ \\
\hline Mediano & $80,84,100,104$ \\
\hline Pequeño & $20,56,76,80$ \\
\hline Muy Pequeño & $0,0,16,20$ \\
\hline
\end{tabular}

El análisis del diagrama de dispersión (Fig5) permite seleccionar para cada atributo (B3, B4), 5 funciones miembro trapezoidales correspondientes a los 5 términos lingüísticos denominados: muy grande $(\mathrm{MG})$, grande $(\mathrm{G})$, mediano $(\mathrm{M})$, pequeño (P) y muy pequeño (MP). En la Tabla I, aparecen definidas las funciones miembro para cada atributo. Mediante el método descrito anteriormente y supervisando las firmas espectrales de las diferentes clases (Fig6) se han seleccionado 315 patrones de entrenamiento.

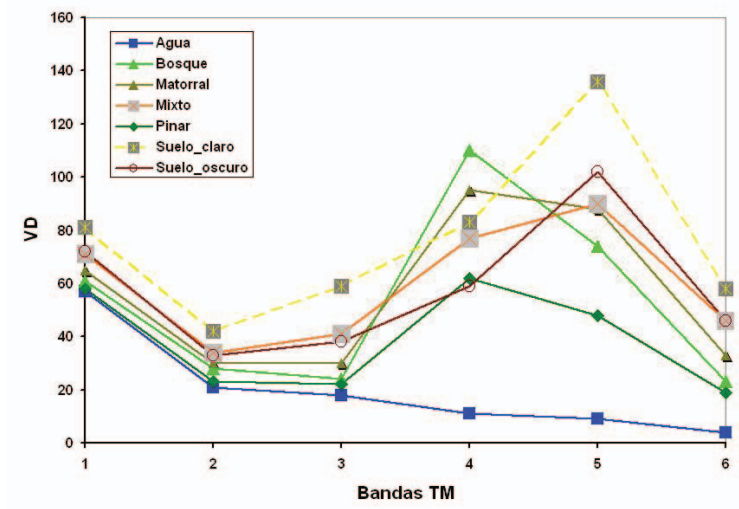

Figura 6. Valor medio (Valor Digital) de las firmas espectrales de los patrones de entrenamiento para las siete clases seleccionadas. 


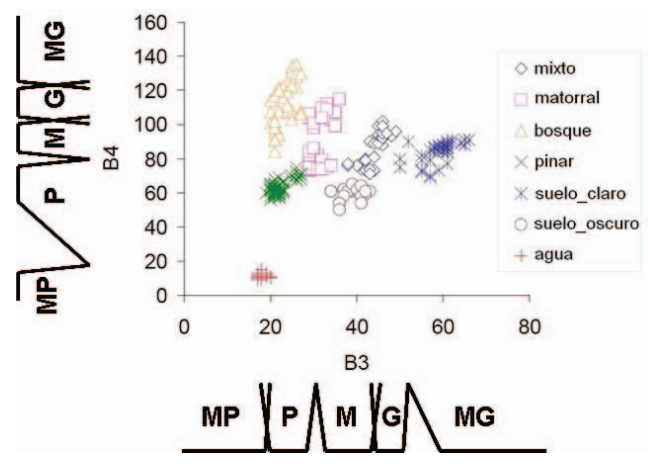

Figura 7. Diagrama de dispersión 2D (B3/B4) de las áreas de entrenamiento junto con la definición de los términos lingüísticos y las funciones miembro.

En la Fig7 se representan los valores especificados en la Tabla I, que dividen el dominio espectral bidimensional de entrenamiento mediante trapecios no regulares.

En la Tabla II se muestra el conjunto de las reglas obtenidas, mediante la metodología basada en algoritmos genéticos descrita en la sección anterior, para el caso de $\alpha$ y $\beta$ igual a 0.75 y $\delta$ igual a 0.1 .

\section{TABLAa II. CONJUNTO ÓPTIMO DE REGLAS DIFUSAS}

\begin{tabular}{|l|}
\hline \multicolumn{1}{|c|}{ REGLAS $\left(\mathbf{R}_{\mathbf{i}}\right)$} \\
\hline R1: IF B3=muy pequeño, B4=pequeño THEN pinar 1,$0000 ;$ \\
\hline R2: IF B3=pequeño, B4=pequeño THEN pinar 1,$0000 ;$ \\
\hline R3: IF B3=muy grande, B4=pequeño THEN suelo claro 1,$0000 ;$ \\
\hline R4: IF B3=muy grande, B4=medio THEN suelo claro 1,$0000 ;$ \\
\hline R5: IF B3=grande, B4=medio THEN mixto 1,$0000 ;$ \\
\hline R6: IF B3=medio, B4=pequeño THEN matorral 1,0000; \\
\hline R7: IF B3=medio, B4=medio THEN matorral 1,0000; \\
\hline R8: IF B3=medio, B4=pequeño THEN suelo oscuro 1,$0000 ;$ \\
\hline R9: IF B3=grande, B4=pequeño THEN suelo oscuro 1,0000; \\
\hline R10: IF B3=pequeño, B4=medio THEN bosque 1,$0000 ;$ \\
\hline R11: IF B3=muy pequeño, B4=muy pequeño THEN agua 1,$0000 ;$ \\
\hline R12: IF B3=pequeño, B4=muy pequeño THEN agua 1,$0000 ;$ \\
\hline
\end{tabular}

Si se analiza el conjunto de reglas obtenidas, solamente las clases mixto y bosque caduco están representadas por una única regla, mientras que las demás clases están representadas por dos reglas. Finalmente, la escena ha sido clasificada (Fig8) mediante inferencia a partir de los patrones de entrenamiento (315 píxeles), y del conjunto de reglas. Además de las siete clases contempladas en la fase de selección de los patrones de entrenamiento, el clasificador ha dado un conjunto de píxeles (3076) agrupados en la clase denominada no clasificados que representan el $2.08 \%$ del total de píxeles de la imagen.

Para llevar a cabo la verificación de los resultados de clasificación se han utilizado un conjunto de patrones test, a partir de los cuales se ha generado la correspondiente matriz de confusión [21], calculándose los errores por omisión y comisión para cada clase temática, la fiabilidad global (FG), y el estadístico kappa (K). Los parámetros de error para la imagen clasificada se recogen en la Tabla III. Un análisis de los resultados, indica que las clases con mayores errores son las denominadas: mixto, matorral y suelo oscuro, clases que presentan una alta variabilidad espectral. Sin embargo K presenta un nivel de concordancia alto $(0.92)$ y la FG resulta ser del $96.19 \%$, valores muy satisfactorios si se contrastan con los obtenidos por Mitrakis et al. [14] en la clasificación de zonas agrícolas mediante un clasificador clásico paramétrico como es el de Máxima Verosimilitud y mediante un clasificador Neurodifuso propuesto para mejorar los resultados.

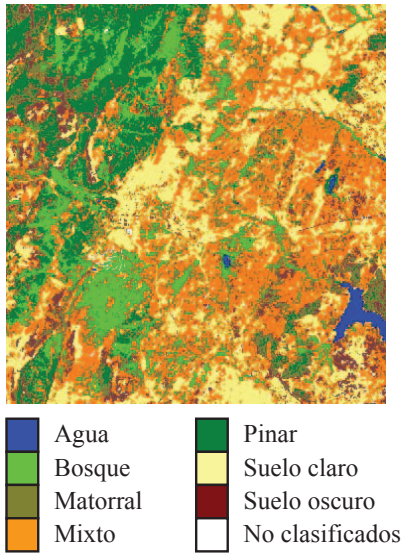

Figura 8. Imagen clasificada.

TABLA III. PARÁMETROS DE ERROR DE LA IMAGEN CLASIFICADA

\begin{tabular}{|c|c|c|}
\hline CLASES & $\begin{array}{c}\text { ERROR POR } \\
\text { OMISION (\%) }\end{array}$ & $\begin{array}{c}\text { ERROR POR } \\
\text { COMISION (\%) }\end{array}$ \\
\hline Agua & 0 & 0 \\
\hline Bosque & 0 & 5.26 \\
\hline Matorral & 4.17 & 8.33 \\
\hline Mixto & 6.67 & 3.33 \\
\hline Pinar & 1.94 & 0 \\
\hline Suelo claro & 0 & 0 \\
\hline Suelo oscuro & 8.00 & 96.19 \\
\hline \multicolumn{2}{|r|}{ FIABILIDAD GLOBAL } & 0.92 \\
\hline \multicolumn{2}{|r|}{ COEFICIENTE KAPPA } & \\
\hline
\end{tabular}

\section{CONCLUSIONES}

Se ha propuesto una metodología basada en lógica difusa, para la clasificación de imágenes multiespectrales registradas mediante sistemas de Teledetección. Además, se ha mostrado que la aplicación de un algoritmo genético permite la determinación objetiva y automática de un conjunto de reglas difusas óptimo que constituyen la base de conocimiento para el sistema clasificador.

Se ha probado la capacidad de los diagramas de dispersión de bandas no correladas para la definición de los términos lingüísticos.

Una de las principales ventajas que ofrece la modelización difusa es la flexibilidad en la definición de diferentes funciones para un mismo término referido a distintos atributos, es decir, desde el punto de vista semántico existe una clara diferencia entre los valores medios en la Banda 3 (atributo 1) y un valor medio en la Banda 4 (atributo 2), y el modelo es capaz de contemplar y representar este matiz.

Los parámetros de error estimados para los resultados de clasificación de la imagen han resultado muy satisfactorios, siendo además preciso indicar que el porcentaje de píxeles considerados como no clasificados es muy bajo $(2.08 \%$ del total de píxeles de la imagen). Dado que estos píxeles están 
dispersos dentro de la imagen, no se puede considerar que constituyan una nueva clase temática significativa.

\section{REFERENCIAS}

[1] J.R. Parker, Algorithms for Image Processing and Computer Vision, Ed. John Wiley \& Sons Inc., New York, 1997.

[2] M. Song, D.L. Civco and J.D. Hurd, (2005).

"A competitive pixel-object approach for land cover classification" International Journal of Remote Sensing 26 (22), pp. 4981-4997.

[3] J. A. Richards, Remote sensing Digital Image Analysis. An introduction, Ed. Springer-Verlag, 1995.

[4] X.H. Liu, A.K. Skidmore and H.V. Oosten, (2002), "Integration of classification methods for improvement of land-cover map accuracy". ISPRS Journal of Photogrammetry and Remote Sensing 56 (4), pp.257268.

[5] D. Lu and Q. Wen, (2007), "A survey of image classification methods and techniques for improving classification performance". International Journal of Remote Sensing 28 (5), pp. 823-870.

[6] A.K. Skidmore, (1989). "An expert system classifies eucalypt forest types using Thematic Mapper data and a digital terrain model". Photogrammetric Engineering and Remote Sensing 55 (10), pp. 14491464.

[7] E. Martínez, C. Gonzalo, A. Arquero and O. Gordo, "Evaluation of different Fuzzy knowledge acquisition methods for remote sensing image classification," in Proc. 1999 IEEE Geoscience and Remote Sensing International Symposium, pp. 2498-2491.

[8] B. Kosko, Neural Networks and Fuzzy Systems. A Dynamical System Approach to Machine Intelligence, Ed. Prentice Hall, 1991

[9] R. Fageth, W.G. Allen and U. Jager, (1996), "Fuzzy logic classification in image processing", Fuzzy set and systems 82, pp. 295-304.

[10] M. Azul Gonzalez y V. Laura Ballarin, (2008), "Image segmentation using Watershed Transform: Marker definition based on fuzzy logic", IEEE Latin America Transactions, 6(2), pp. 223-228.

[11] H. Ishibuchi, K. Nozaki, N. Yamamoto and H. Tanaka, (1994), "Construction of fuzzy classification systems with rectangular fuzzy rules using genetic algorithms", Fuzzy set and systems 65, pp. 237-256.

[12] M. H. Tseng, S. J. Chen, G. H. Hwang and M.Y. Shen, (2008), "A genetic algorithm rule-based approach for land-cover classification", ISPRS Journal of Photogrammetry \& Remote Sensing 63, pp. 202-212.

[13] D. Stathakis and A. Vasilakos, 2006, "Comparison of Computational Intelligence Based Classification Techniques for Remotely Sensed Optical Image Classification", IEEE Transactions on Geoscience and Remote Sensing, 44 (8), pp. 2305-2318.

[14] N. E. Mitrakis, C. A. Topaloglou, T. K. Alexandridis, J. B. Theocharis, and G. C. Zalidis, (2008), "Decision Fusion of GA Self-Organizing Neuro-Fuzzy Multilayered Classifiers for Land Cover Classification Using Textural and Spectral Features", IEEE Transactions on Geoscience and Remote Sensing, 46 (7), pp. 2137-2151.

[15] M. A. Muñoz, J.A. López y E. F. Caicedo, (2008), "Inteligencia de enjambres: sociedades para la solución de problemas (una revisión)", Revista Ingeniería e Investigación 28 (2), pp. 119-130.

[16] A. Jubai, B. Jing and J. Yang, (2006), "Combining fuzzy theory and a genetic algorithm for satellite image edge detection", International Journal of Remote Sensing, 27(14), pp. 3013-3024.

[17] Y. Yuan and H. Zhuang, (1996), "A genetic algorithm for generating fuzzy classification rules", Fuzzy set and systems, 84, pp.1-19.

[18] A. Arquero, C. Gonzalo, E. Martínez and A. Ferreras, (1998), "Utilización de Scattergrams de ER Mapper para la elección de las áreas de entrenamiento en la clasificación temática de imágenes Landsat" Mapping. Revista de Cartografía, Sistemas de Información Geográfica, Teledetección y Medio Ambiente 50, pp. 56-58.

[19] Cuena J. Notas sobre modelos de razonamiento. Facultad de Informática, Universidad Politécnica de Madrid, Madrid, 1997.

[20] E. Martinez, C. Gonzalo, A. Arquero y M. Lillo, "Evaluation of a Change Detection Method based on joint spatial-spectral Information" in Remote Sensing In Transition. Proc. EARSEL 2004 pp. 121-126.

[21] E, Chuvieco. Teledetección Espacial, Ed. Ariel Ciencia. Madrid, 2002.

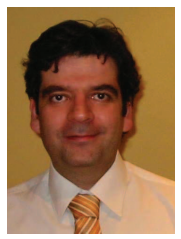

Óscar Gordo Martín, obtuvo el título Licenciado en Informática por la Universidad Politécnica de Madrid (UPM) en 1998. Actualmente desarrolla las investigaciones correspondientes al desarrollo de su Tesis Doctoral dentro del ámbito de las líneas de investigación del Grupo de Teledetección de la Facultad de Informática de la UPM (España). Entre dichas investigaciones destacan la definición y aplicación de procesos de Ingeniería del Software y arquitecturas software abiertas al desarrollo de sistemas de procesado de imágenes con aplicación a la Teledetección.

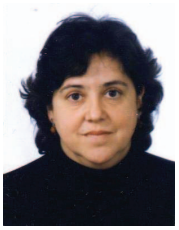

Estíbaliz Martínez Izquierdo es Doctora en Ciencias Químicas por la Universidad Complutense de Madrid (España) desde julio de 1989. Realizó su Tesis Doctoral en el Análisis y Procesado de Información Espectral. Actualmente es Prof. Titular en el Dpto. de Arquitectura y Tecnología de Sist. Informáticos de la Facultad de Informática de la UPM (España). Imparte docencia en Diseño Digital, VLSI y Proc. de Imagen y Teledetección. Su investigación está en las áreas del procesado y análisis de imágenes en Teledetección, desarrollando nuevos algoritmos para segmentación, clasificación y detección de cambios, incluyendo los basados en Redes Neuronales, Genéticos y Lógica Difusa.

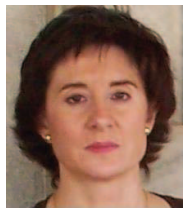

Consuelo Gonzalo Martín, obtuvo el título de Licenciada en Ciencias. Físicas por la Universidad de Salamanca en 1986. Realizó su Tesis Doctoral en Procesado Óptico y Digital de Imágenes en el Ins. de Óptica del CSIC. Se doctoró en Ciencias Físicas en la U. Complutense de Madrid en 1989. Desde entonces es profesora en el Dpto. de Arquitectura y Tecnología de Sist. Informáticos de la UPM (España). Su área de investigación es el desarrollo de algoritmos de procesado de imágenes con aplicación en Teledetección y de una forma particular en algoritmos de fusión de imágenes de satélite.

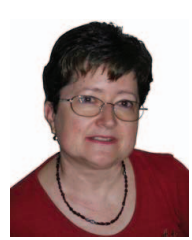

Águeda Arquero Hidalgo, obtuvo el grado de Doctor en Ciencias Químicas por la Universidad Autónoma de Madrid en 1983. Actualmente es Prof. Titular de Universidad en el Dpto. de Arquitectura y Tecnología de Sist. Informáticos, en la Facultad de Informática de la UPM. Su investigación en el campo del procesado y análisis de imágenes remotamente detectadas incluye la medición de la respuesta radiométrica de cubiertas terrestres in situ y su procesado posterior; el análisis de datos teledetectados usando algoritmos de RNA, genéticos y lógica difusa. 